\title{
A MODEL OF P/TEMPEL 2 WITH DUST AND DETAILED CHEMISTRY
}

\author{
W.F. HUEBNER ${ }^{1}$, D.C. BOICE ${ }^{1}$, I. KONNO ${ }^{1}$, and P.D. SINGH ${ }^{2}$ \\ ${ }^{1}$ Southwest Research Institute, San Antonio, TX 78228-0510 U.S.A. \\ ${ }^{2}$ Instituto Astronômico e Geofísico, Universidade de São Paulo, \\ C.P. 9638, São Paulo, S.P. Brazil
}

\begin{abstract}
We apply our fluid dynamic model with chemical kinetics of dusty comet comae to $\mathrm{P} /$ Tempel 2. A brief summary of results concerning gas/dust dynamics and chemistry is given.
\end{abstract}

One-dimensional, multi-fluid simulations of the coma of $\mathrm{P} /$ Tempel 2 at perihelion have been performed. These simulations are based on our model that treats the physics and chemistry of the inner coma in great detail as summarized by Schmidt et al. (Comp. Phys. Comm. 49, 17-59, 1988). Recent progress of the model includes incorporation of dust entrainment by the gas, dust size distributions, dust fragmentation, distributed coma sources of gasphase species related to the dust, and a separate accounting of the electron energetics. An improved calculation for the sublimation rate of water from the nucleus is now performed and the chemical reaction network has been expanded to include $\mathrm{CH}_{3} \mathrm{OH}, \mathrm{H}_{2} \mathrm{~S}$, and $\mathrm{H}_{2} \mathrm{CO}_{2}$ as possible parent molecules. The assumed volatile composition is given in Table 1 and other model parameters are listed in Table 2.

The model species profiles are appropriate for neutrals throughout the coma and for ions within the contact surface. Figure 1 illustrates that methanol is a distributed source of $\mathrm{H}_{2} \mathrm{CO}$ upon photodissociation with a lifetime of $1.96 \cdot 10^{4} \mathrm{~s}$ (quiet sun). In turn, $\mathrm{H}_{2} \mathrm{CO}$ forms a distributed source for $\mathrm{CO}$ with a lifetime of $5.15 \cdot 10^{3} \mathrm{~s}$. Several organic species, as well as the dust, contribute to enhance the coma abundance of $\mathrm{CH}$ and extend its range. As shown in Fig. 2, protonated parent molecules may be major ionic species in the inner coma if the parent abundances in the nucleus are $Z 0.5 \%$ with proton affinities greater than that of water (e.g., $\mathrm{NH}_{3}, \mathrm{CH}_{3} \mathrm{OH}, \mathrm{H}_{2} \mathrm{CO}, \mathrm{H}_{2} \mathrm{CO}_{2}$ ). It can be seen that the abundance of a protonated parent species is higher than the respective parent ion in the inner coma $(R \lesssim 500 \mathrm{~km})$. For illustrative purposes, we have set the abundance of $\mathrm{H}_{2} \mathrm{CO}_{2}$ rather high (1\%) in the nucleus composition which leads to its protonated ion $\mathrm{H}_{3} \mathrm{CO}_{2}^{+}$being the most abundant ion in the inner coma. This demonstrates the sensitivity of the abundances of protonated species on the initial composition of these parents.

The dust mass distribution is approximated by a power law (exponent $\beta$ ) with 11 discrete sizes, logarithmically spaced between $a_{\min }$ and $a_{\max }$. The model capabilities for dust fragmentation are not used in the present calculations. Gas and dust are rapidly accelerated upon leaving the nucleus. For standard dust densities, small particles are more efficiently entrained with the gas flow than large particles, resulting in higher terminal speeds. The acceleration zone for all particles is approximately within $10 R_{n u c}$. A complete description of the model and results will be presented in a forthcoming publication.

This research was supported by grants from NASA (Nos. NAGW-2205, NAGW-2370), FAPESP (No. 90/1384-3), CNPq(No. 304076/77), and NSF (No. INT-8901811). 
TABLE 1. Composition of P/Tempel 2 Model

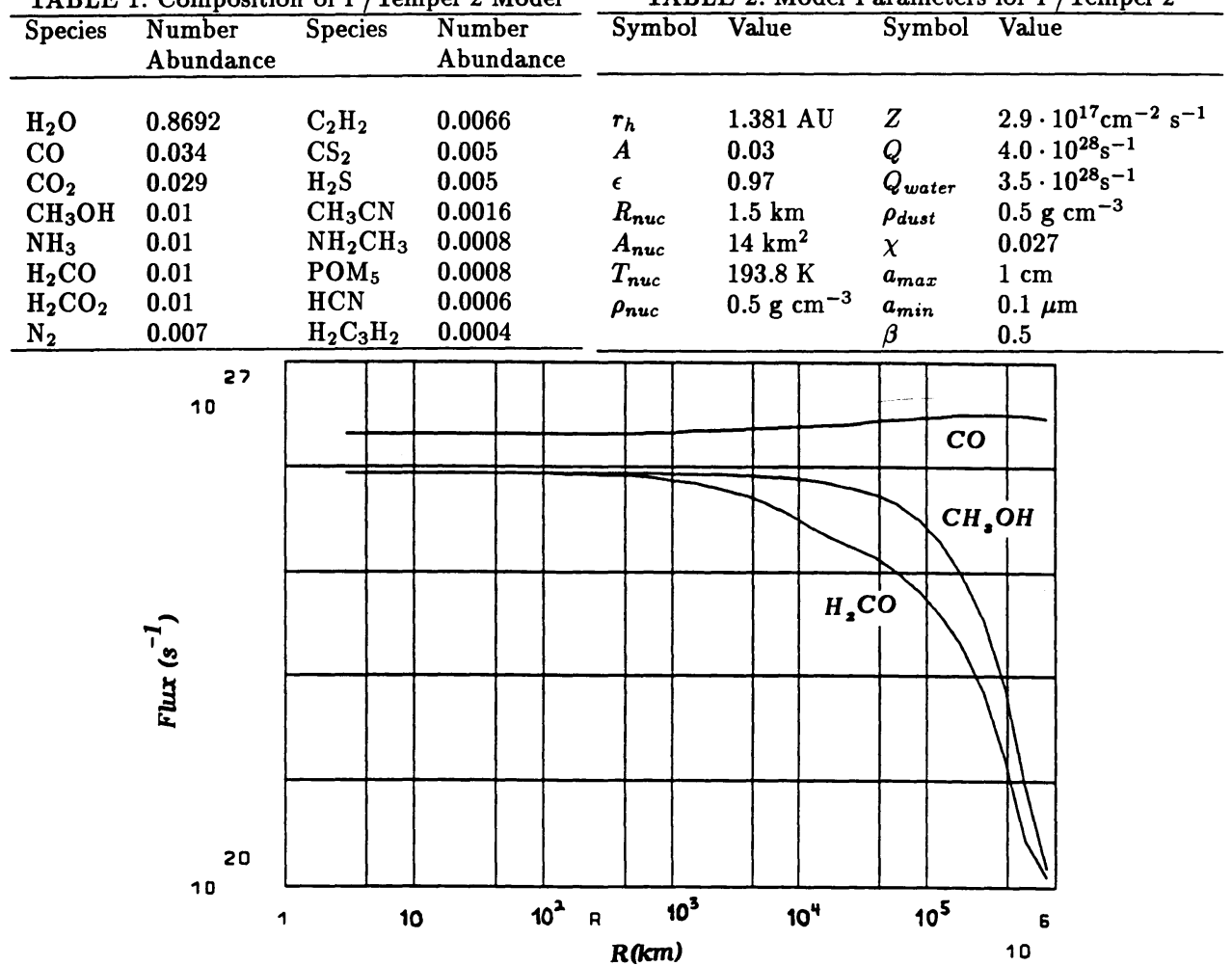

Figure 1. Profiles of the integrated flux for $\mathrm{CO}, \mathrm{CH}_{3} \mathrm{OH}$, and $\mathrm{H}_{2} \mathrm{CO}$ throughout the coma.

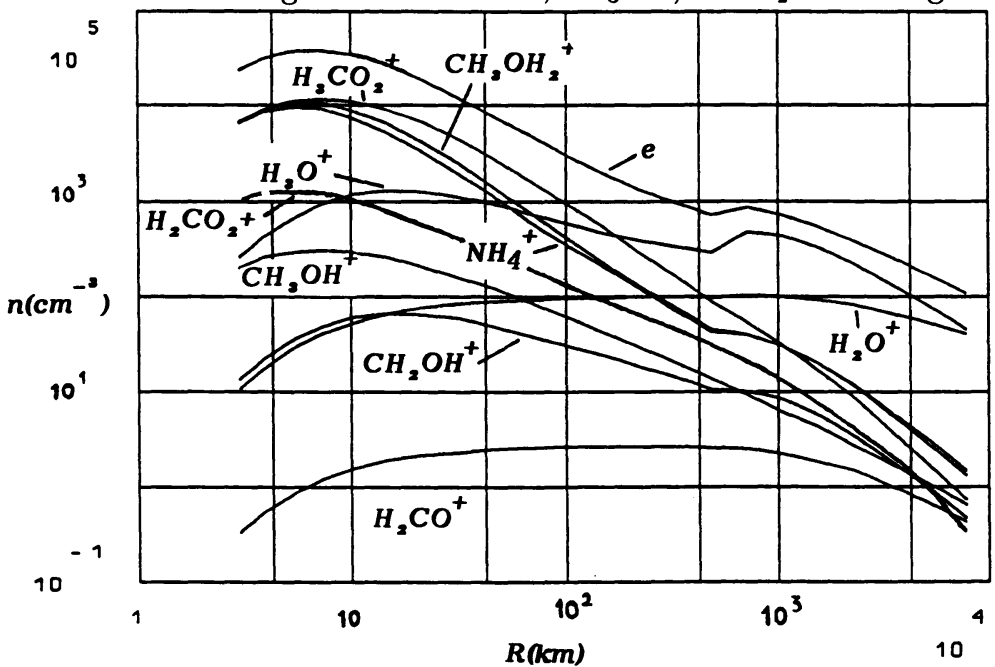

Figure 2. Number density profiles of electrons and ions related to selected parent molecules via protonization and ionization. 